\title{
THE SPREAD OF CURRENTS AND DISTRIBUTION OF POTENTIALS IN HOMOGENEOUS VOLUME CONDUCTORS*
}

\author{
By Franklin Davis Johnston \\ University of Michigan Medical School, Ann Arbor, Mich.
}

Frank N. Wilson struggled for several years during the 'twenties to convince physicians and physiologists that laws governing the flow of currents and the distribution of potentials in volume conductors must be applied to electrocardiography. In 1932 he had already completed a long, basic paper largely devoted to this subject, and this was published as a monograph by the University of Michigan Press the following year. ${ }^{1}$ Several of the figures used to illustrate my brief discussion of the matters under consideration have been taken from that monograph.

FIGURE 1 indicates that A. Waller, in the last century, had a good concept of the distribution of currents and potentials arising from the heart. In this figure, voltages produced by the heart are represented by two poles of a battery $A$ and $B$, and a few of the infinite number of paths of current flow are indicated by the fine dotted lines. The solid lines surrounding pole $A$ and the dashed and dotted lines close to pole $B$ represent regions of equal potential. Thus, if $A$ is the positive and $B$ the negative pole of the battery, all points on each dashed and dotted line are equally positive in potential, and all points on each solid line are equally negative in potential. It may be pointed out that a vector collinear with the current axis through $A$ and $B$ may be used to represent the magnitude and direction of the electromotive force (E.M.F.) of the battery, and that a line drawn perpendicular to the line joining the poles of the battery at its mid-point, that is, the zero potential line, should be an excellent reference point for the measurement of potential anywhere in the medium.

Before the Einthoven triangle concept and subsequent developments are discussed, a few words should be said about some of the mathematical expressions that may be employed to determine the potential of any point in volume conductors of different kinds. Such expressions are available only for homogeneous conductors of simple types, and some of these are shown in FIGURE 2A. EQUATIONS 1 and 2 apply to a plane lamina infinite in extent, and EQUATION 3 applies to a circular lamina of radius $R$. EQuations 4 and 5 apply to a homogeneous medium of infinite extent in all directions, and (6) applies to a spherical medium of radius $R$. For the derivation of these equations, the interested reader is referred to the monograph of Wilson and his associates ${ }^{1}$ mentioned above.

During the $1920 \mathrm{~s}$, when Wilson was working to prove that laws pertaining to the flow of currents in volume conductors must apply to electrocardiography,

* Support for studies presented in this article has been provided by a grant to Frank N. Wilson by the S. S. Kresge Foundation, Detroit, Mich.; and funds have also been provided by the Michigan Heart Association, Detroit, Mich., and the National Institutes of Health, Public Health Service, Department of Health, Education. and Welfare, Bethesda, Md. (HT5114). 


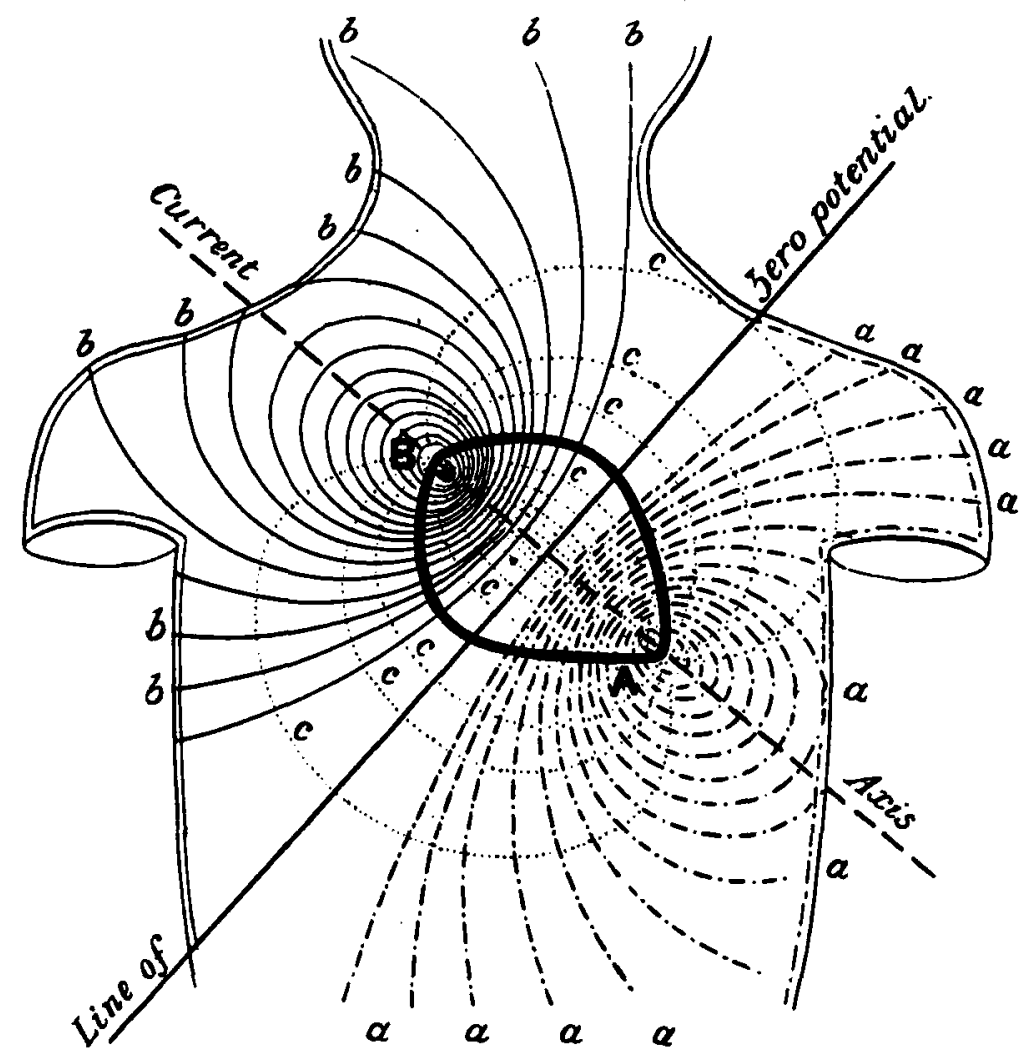

FIgURE 1. $A$ and $B$ are 2 points of the apex and base, respectively. A straight line between $A$ and $B$ represents the axis of current between $A$ and $B$ if any inequality of potential should arise between the two points. The dotted lines $(c)$ represent lines of current diffusion; the broken lines $(a)$ represent equipotential lines surrounding the point $A$; and the continuous lines $(b)$ represent equipotential lines surrounding the point $B$. A straight line at right angles to the current axis represents the line of zero potential.

Craib $^{2-4}$ was engaged in similar studies employing strips of cardiac and skeletal muscle and medullated nerve fibers. The results of Craib's studies were published before Wilson had completed all of his work, but the opinions of the two workers on opposite sides of the Atlantic Ocean were in harmony. In this connection, Wilson" wrote: "In stating that work on the general subject of this monograph was begun in this laboratory a number of years before Craib's first paper was published (see Wilson, Wishart and Herrmann, $1926,{ }^{5}$ and Wilson, $1930^{6}$ ) and that many of the facts to which he has called attention were known to one of us long before that paper appeared, we do not wish to raise any question of priority or in any way to claim any share of the credit due Craib for the fine work he has done. Inasmuch as our work has been done independently of his, we have naturally followed our own point of view. Craib's work has, however, made it unnecessary for us to carry out experiments 
A

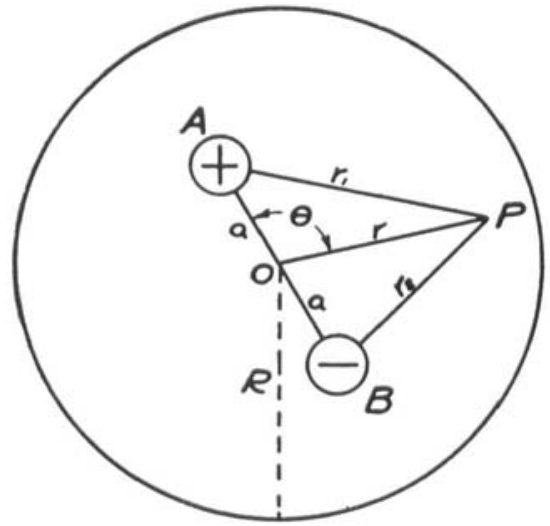

$$
\begin{array}{lll}
V=c \log \frac{r_{2}}{r_{1}} & \text { (1) } & V=c^{\prime}\left(\frac{1}{r_{1}}-\frac{1}{r_{2}}\right) \\
r=2 a c \frac{\cos \theta}{r} & \text { (2) } & V=2 a c^{\prime} \frac{\cos \theta}{r^{2}} \\
V=2 a c \cos \theta\left(\frac{1}{r}+\frac{r}{R^{2}}\right) & \text { (3) } & V=2 a c^{\prime} \cos \theta\left(\frac{1}{r^{2}}+\begin{array}{l}
2 r \\
R^{2}
\end{array}\right)
\end{array}
$$

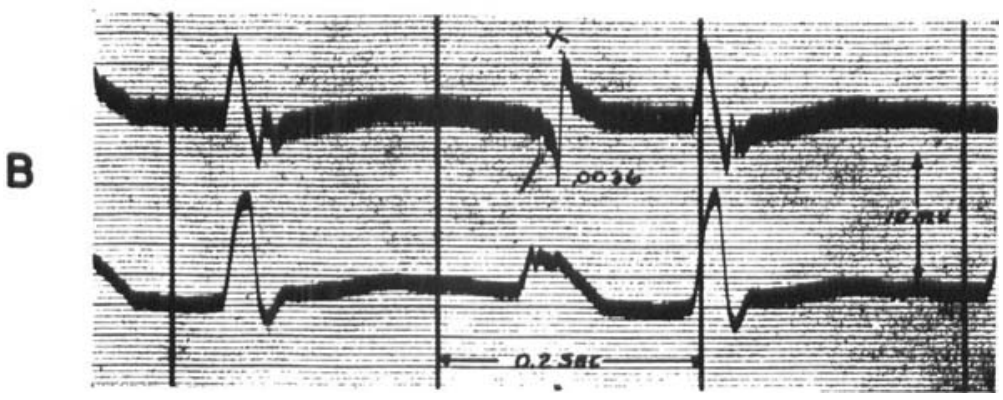

FigURE 2. In FIGURE $2 B$ the to $p$ curve represents a direct lead from a point on the surface of the auricle of a large dog, midway between the upper end of the sulcus terminalis and the tip of the right auricular appendix. An upward deflection indicates the relative negativity of the exploring electrode. The indifferent electrode is on the left hind leg. Ten mv. equals $2 \mathrm{~cm}$. The deflection time of the string for $10 \mathrm{mv}$, is about $0.0028 \mathrm{sec}$. There is slight overshooting, and the string is not quite aperiodic. The duration of the intrinsic auricular deflection is about 0.0036 (Lucas comparator). The lower curve represents the exploring electrode near the junction of the superior vena cava and the right auricle, possibly on the vein. The indifferent electrode is on the right hind leg. (Reproduced by permission of the University of Michigan Press, Ann Arbor, Mich.)

upon simple tissue strips or to enter at length upon subjects that have been adequately discussed in his papers."

It is clear from the above that Wilson was in full agreement with the work Craib had done with muscle strips and other simple preparations, and it remained only to prove that laws governing the flow of currents in volume conductors applied equally to the mammalian heart beating in the experimental animal. To accomplish this, essentially unipolar electrocardiograms (FIGURE 2B) were recorded midway from the upper end of the sulcus terminalis to 
the tip of the right auricular appendix of a large dog. It was then shown that curves obtained from mathematical expressions derived from the basic equations of FIGURE 2A were almost identical to the complexes, in the direct lead, that represented accession in the auricular muscle.

Only two of the expressions derived by Wilson and his associates ${ }^{1}$ will be mentioned here. The first is

$$
V=C^{\prime}\left(\frac{1}{\sqrt{(x-a)^{2}+b^{2}}}-\frac{1}{\sqrt{(x+a)^{2}+b^{2}}}\right)
$$

which applies to the simple situation illustrated in FIGURE 3A. Here the 2 poles of a dipole separated by a distance $2 a$ are assumed to be moving at a uniform velocity along the $X$ axis from left to right. $V$ is the potential at any point $P$ located at a distance $b$ from the $X$ axis. If the arbitrary values for $C^{\prime}, a$, and $b$ are used in the above equation, the curve that it represents is shown in FIGURE 3B. It will be observed that this curve closely resembles the complex in FIGURE 2B (marked $X$ ) obtained from the electrode on the auricular muscle.

A more complicated case, but one that represents the actual conditions in the auricle quite closely, is illustrated in FIGURE 4A. The equation representing the potential at any point $P$ and the corresponding curve are shown in FIGUREs $4 \mathrm{~B}$ and $4 \mathrm{C}$. It will be noticed that this curve is very much like the complex marked $X$ in FIGURE 2B.

Let us now return to the Einthoven triangle. This concept relates records taken with electrodes on the extremities to electrical events in the heart; although its inaccuracies have been recognized for a long time, it has been, and probably will continue for many years to be, valuable in clinical electrocardiography. FIGURE 5A illustrates the ideas involved and shows graphically the relations between a voltage $O E$ acting in the heart and what is recorded in the standard limb leads. FIGURE 5B gives an algebraic statement of voltages in the limb leads in terms of the vector $p q$ or $E$ and the angle alpha $(\alpha)$. Underlying the equilateral triangle idea is the assumption that the body can be represented by a homogeneous conducting medium of large extent. It does not matter whether one assumes this to be a triangle, a circular disc, an infinite lamina, or a sphere of large or infinite radius, provided the three electrodes are symmetrically located about a centrally placed equivalent dipole whose positive and negative poles are close together compared to the equal distances between the center of the dipole and the three electrodes.

It should be pointed out that the vectors $O E$ and $E$ in FIGUREs $5 \mathrm{~A}$ and $5 \mathrm{~B}$ may be considered either the summation of all cardiac E.M.F.s over a period of time (for example, the vector representing all cardiac E.M.F.s during the QRS interval, that is, the mean electrical axis of QRS) or the summation of all voltages acting in the heart at any instant of time during a period of cardiac activity. FIGURE 6 shows the mean instantaneous electrical vectors at intervals of $0.01 \mathrm{sec}$. during the inscription of the QRS complex $\left(\mathrm{O}_{1}, \mathrm{O}_{2}, \mathrm{O}_{3}\right.$, etc. $)$ and the mean electrical axis of QRS $(O E)$. If the tips of all of the former are joined by a smooth curved line, one has the vectorcardiogram that represents, 


\section{Johnston: Homogeneous Volume Conductors}

in a different way, the same information seen in the scalar leads 1,2 , and 3 . It should be emphasized that the limb leads, the Einthoven triangle arrangement, and the vectorcardiogram shown in FIGURE 6 are concerned with components of cardiac voltages in the frontal plane.

Supposing that the assumption underlying the Einthoven triangle is strictly true, Wilson and his associates ${ }^{7}$ showed in the early 1930s that a network by
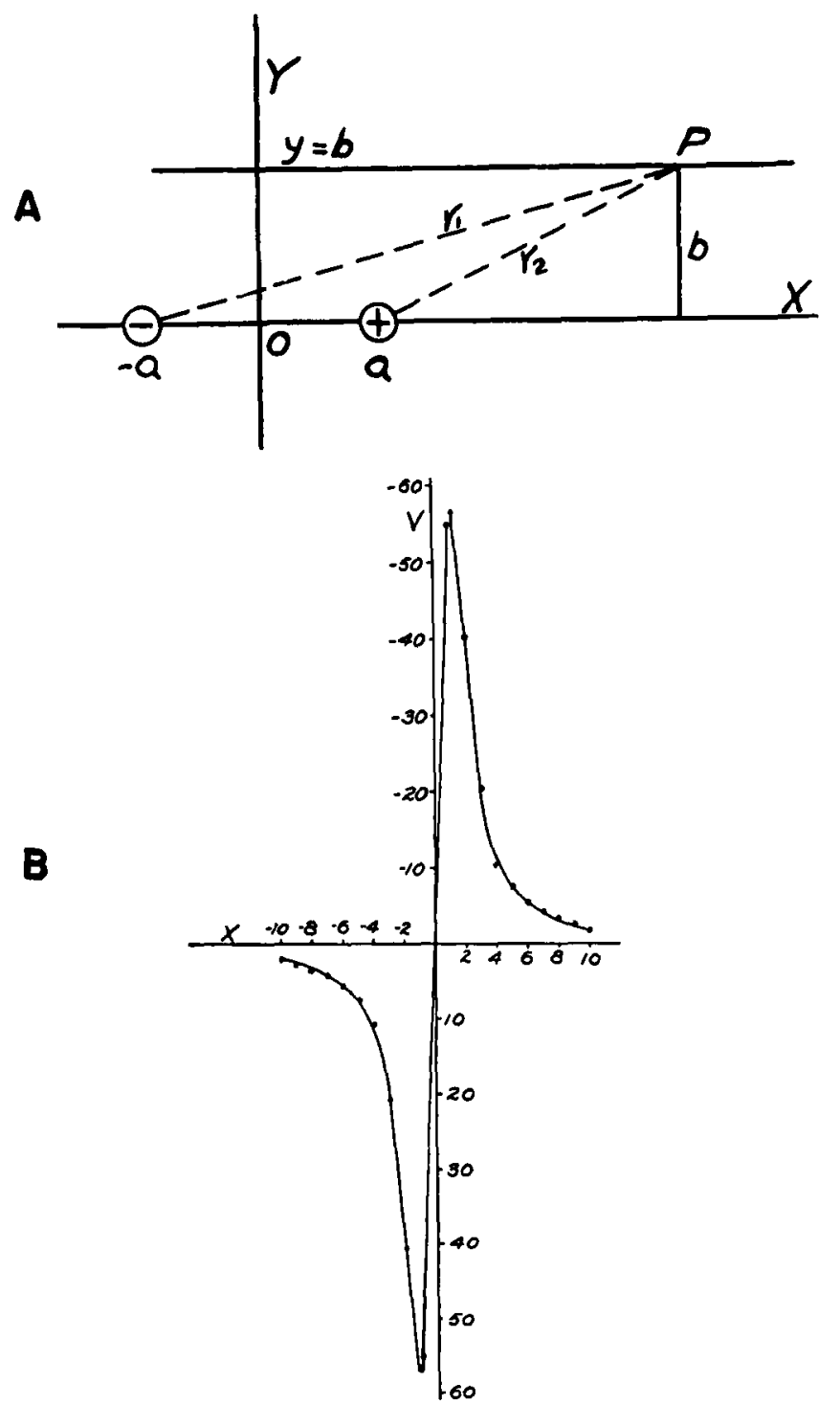

Figure 3. Reproduced by permission of the University of Michigan Press, Ann Arbor, Mich. 
which the electrodes on the right arm, left arm, and left leg are connected to a common point through equal resistances of at least 5000 ohms established an indifferent electrode with small potential variations throughout the cardiac cycle. Figure 7A shows this scheme in diagrammatic form. Wilson fully appreciated the inaccuracies of the central-terminal electrode, but he believed that it served as the best indifferent electrode available for the registration of

A

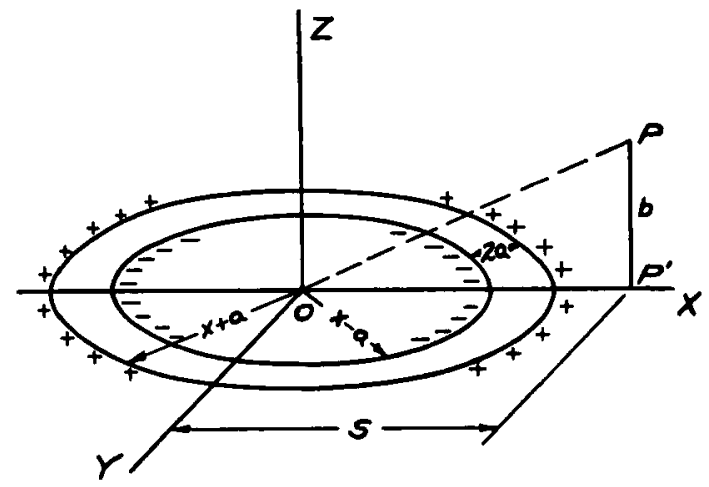

$\mathbf{B}$

$$
r=\frac{4 c^{\prime} \mu(x+a)}{\sqrt{(x+a+s)^{2}+b^{2}}} \int^{\frac{\pi}{2}} \frac{d \phi}{\sqrt{1-k_{1}^{2} \sin ^{2} \phi}}-
$$

$$
\frac{4 c^{\prime} \mu(x+a)}{\sqrt{(x-a+s)^{2}+b^{2}}} \int_{0}^{\frac{\pi}{2}} \frac{d \phi}{\sqrt{1-k_{2}^{2} \sin ^{2} \phi}}
$$

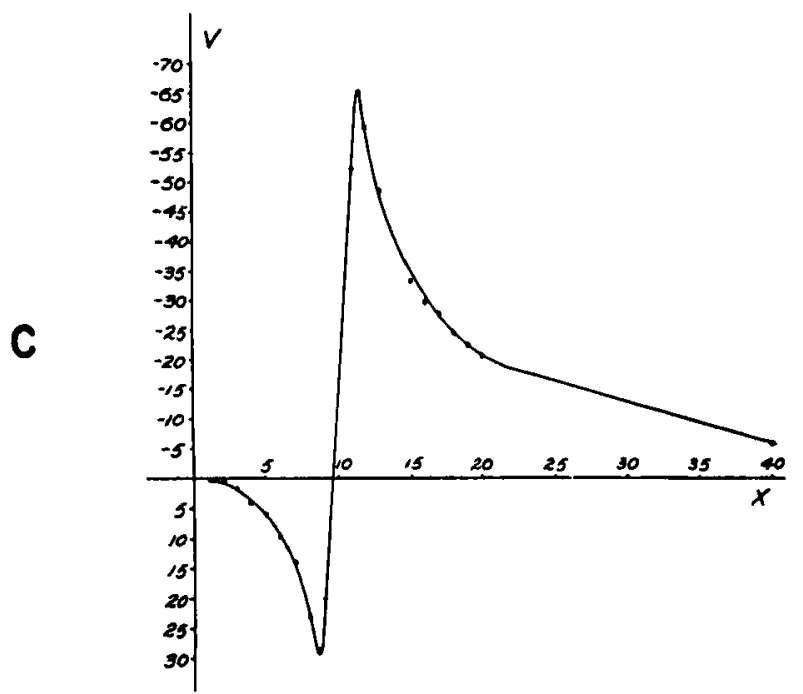

Figure 4. Reproduced by permission of the University of Michigan Press, Ann Arbor, Mich. 
Johnston: Homogeneous Volume Conductors
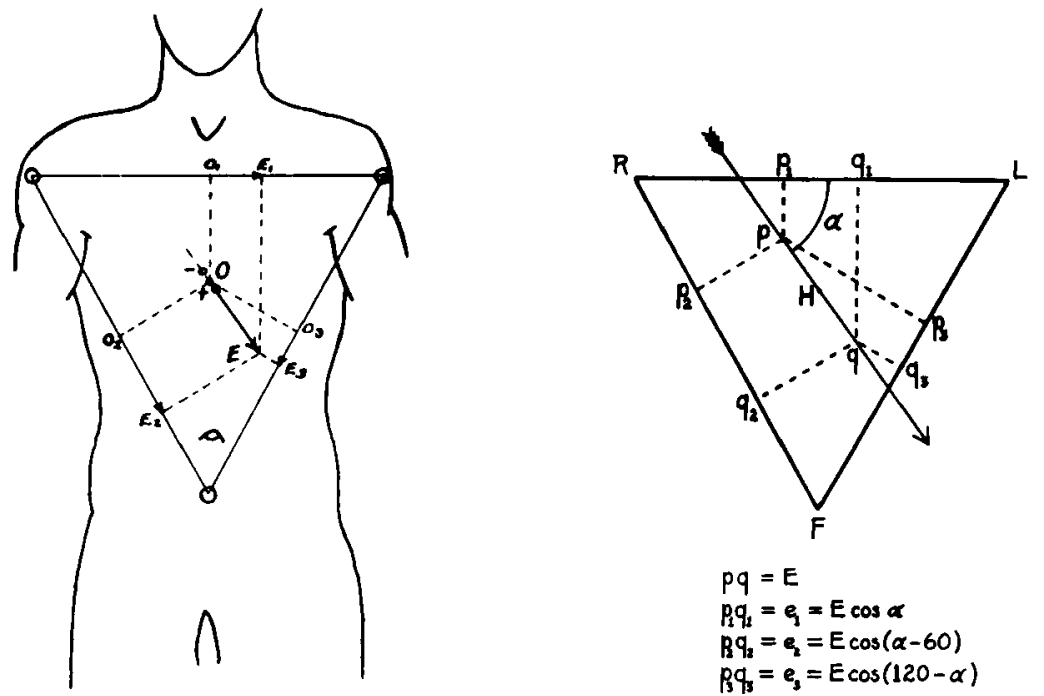

$$
\begin{aligned}
& P q_{1}=E \\
& P_{1} q_{1}=e_{1}=E \cos \alpha \\
& P_{2}=e_{2}=E \cos (\alpha-60) \\
& P_{3} q_{3}=e_{3}=E \cos (120-\alpha)
\end{aligned}
$$

A

B

FigURE 5

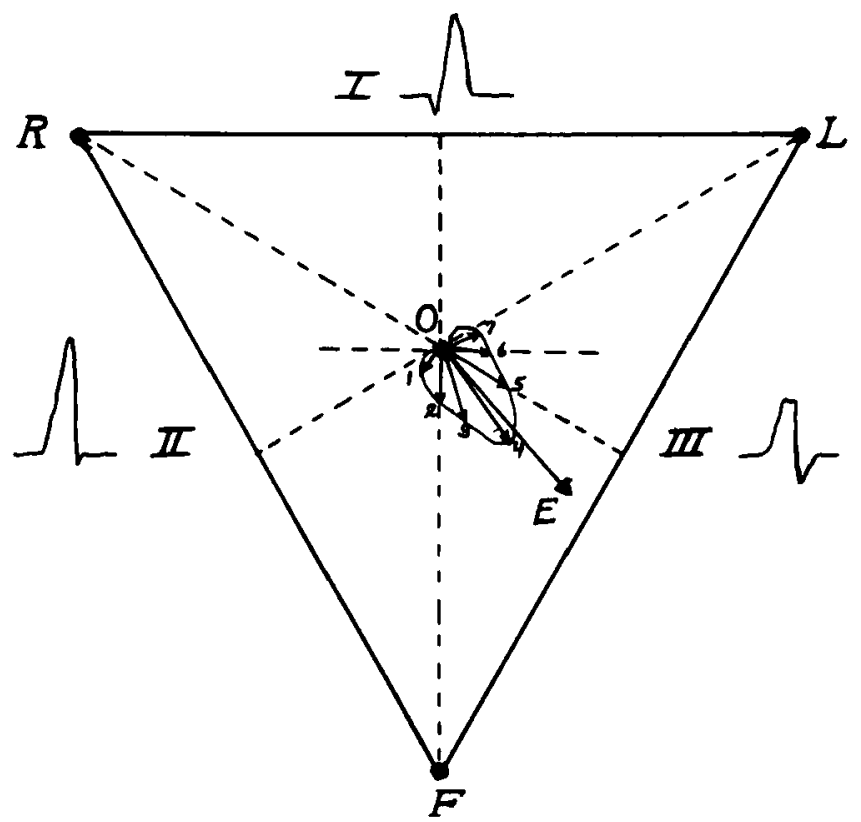

FIgURE 6. Reproduced by permission of W. B. Saunders Co., Philadelphia, Pa. 


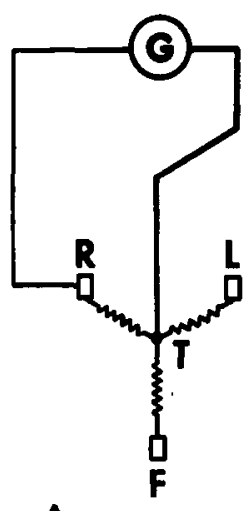

$\mathbf{A}$

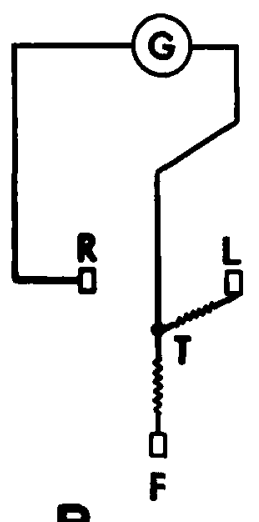

B

Figure 7. Reproduced by permission of F. A. Davis Co., Philadelphia, Pa.

nearly unipolar precordial leads and, further, that it provided an electrode with such small voltage changes that it could be used to obtain tracings that represented, approximately at least, the potential variations of the extremities. A few years later Goldberger ${ }^{8}$ pointed out the modification of the central-terminal circuit needed to take augmented unipolar extremity leads (FIGURE 7B) $\left(a V_{R}, a V_{L}\right.$, and $\left.a V_{F}\right)$ and, thereafter, so-called unipolar electrocardiography assumed the place it holds today.

I have often wondered if Wilson was not somewhat surprised at the nearly universal acceptance of the central-terminal technique. In any event, he fully appreciated and welcomed the lead-vector concept when it was first described by Burger and van Milaan ${ }^{9,} 10$ about ten years ago. This idea is very important, since it provides a tool for the determination of the behavior of any lead, free of most of the restrictions imposed by the assumption that underlies the Einthoven triangle.

Since the lead vector has served as the basis for much important work in the last few years and has been perhaps a somewhat difficult concept for the average physician, a few words describing it are in order. In FIGURE 8A the voltages in the heart are represented by a single equivalent dipole, the size and direction of which are represented by the vector $E$. This vector may be replaced, of course, by its three orthogonal components $x, y$, and $z$. Suppose vector $E$ has a purely transverse direction, in which case the $y$ and $z$ components are zero. Under these circumstances the voltage produced by $E$ (or $x$ ) in any bipolar lead (for example, lead 1 ) is $A x$, where $A$ is a constant or coefficient that involves matters such as inhomogeneity of the tissues and the spatial relations between the E.M.F. $(E x)$ and the electrodes of the lead employed. Similarly, if the direction of $E$ is purely vertical the transverse and sagittal components $x$ and $z$ are zero, and the lead voltage $V$ is $B y$, where $B$ is again a constant coefficient that describes the reaction of the lead in question to a vertically oriented voltage located at a specified point in the heart. If these conditions are true, by the superposition theorem the lead voltage $V$ is equal to $A x+B y+C z$. Burger and van Milaan further pointed out that the coefficients $A, B$, and $C$ may be considered as the $x, y$, and $z$ components of a 


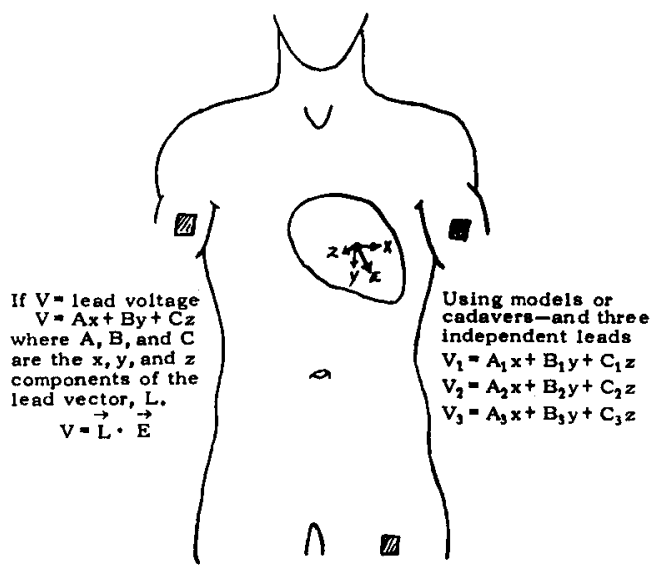

A
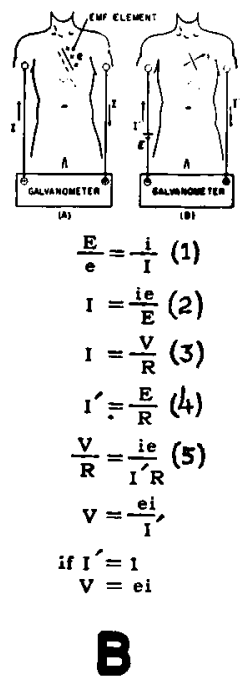
N. Y.

Figure 8. Figure 8B is reproduced by permission of Grune and Stratton, Inc., New York,

vector, which is the lead vector $L$, and this fully describes the performance of a particular lead. It should be emphasized that, for a given lead, determination of the coefficients $A, B$, and $C$ establishes the behavior of the lead for only a single position of the equivalent dipole in the heart. If the dipole is moved these coefficients may change appreciably. Burger and van Milaan also showed that the lead voltage $V$ equals the scalar product of the lead vector $L$ and the vector $E$ that represents the equivalent dipole in the heart. Thus $V$ equals $\vec{L} \cdot \vec{E}$.

It is not possible to estimate the coefficients that establish lead vectors directly in man, but Burger and van Milaan and many other workers more recently, by the use of models or of cadavers, have determined them for many commonly used leads in approximate fashion. This is possible since voltages of known size and direction can be introduced into the cardiac area of the model and, if the voltage produced in a lead is measured with the dipole oriented successively in the $x, y$, and $z$ directions, 3 equations with only 3 unknowns, $A, B$, and $C$, are available, and these can be solved for the coefficients. Thus in the equation $V_{1}=A x$, where $V_{1}$ is the lead voltage, the dipole of known strength is oriented in a strictly transverse manner. Under such circumstances $y$ and $z$ are zero, and the general equation reduces to $V_{1}=A x$. Orientation of the dipole in the vertical and sagittal directions gives the equations $V_{2}=B y$ and $V_{3}=C z$ and allows calculation of $B$ and $C$. If the lead vectors are known for 3 independent leads either in models or man, the size and direction of the equivalent cardiac dipole can be calculated. Thus the equations

$$
\begin{aligned}
& V_{1}=A_{1} x+B_{1} y+C_{1} z \\
& V_{2}=A_{2} x+B_{2} y+C_{2} z \\
& V_{3}=A_{3} x+B_{3} y+C_{3} z
\end{aligned}
$$


where $V_{1}, V_{2}$, and $V_{3}$ are known lead voltages, and $A_{1}, B_{1}, C_{1}$, etc. are the known lead vector coefficients for the respective leads, can be solved for $x, y$, and $z$, the 3 orthogonal components of the vector $E$.

The concept of the lead field has been described in detail by McFee and Johnston. ${ }^{11-13}$ It is not an entirely new concept, since the tubes of influence mentioned by Lepeschkin ${ }^{14}$ represent essentially the same idea. The lead field depends entirely on the reciprocity theorem of Helmholtz; consequently it is essential that this theorem be clearly understood. In his derivation of the reciprocity theorem, Helmholtz was considering the effect of an electromotive surface in a volume conductor on the deflection of a galvanometer connected to that conductor. He stated the theorem in the following way: ${ }^{15}$ "Every single element of an electromotive surface will produce a flow of the same quantity of electricity through the galvanometer as would flow through that element itself if its electromotive force were impressed on the galvanometer wire. If one adds the effects of all the electromotive surface elements, the effects of each of which are found in the manner described, he will have the value of the total current through the galvanometer."*

Referring to FIGURE $8 \mathrm{~B}$, an element of the electromotive surface in the heart, $e$, will produce the same current in the external galvanometer circuit as would flow through the element $e$ were its voltage impressed on the galvanometer circuit. This reciprocal relationship is expressed in more general terms by the equation $\frac{E}{e}=\frac{i}{I}$ (EQUATION 1 in the figure), where $e$, as before, is the voltage of the element in the heart, $E$ is the voltage impressed on the galvanometer circuit, $i$ is the current through the element in the heart, and $I$ is the current in the galvanometer circuit. Equation 2, $I=\frac{i e}{E}$, is EQUATION 1 rearranged and solved for $I$. By Ohm's law the current $I$ in the galvanometer circuit equals the lead voltage $V$ divided by the total resistance between the 2 electrodes $\mathrm{R}$. Thus equation 3 is $I=\frac{V}{R}$. If another battery of voltage $E$ is impressed in the galvanometer circuit it is clear that the current in the galvanometer circuit now becomes Equation $4, I^{\prime}=\frac{E}{R}$. If the values for $I$ in EQUATION 3 and for $E$ in EQUATION 4 are substituted in EQUATION 2, the following expression is obtained: $\frac{V}{R}=\frac{i e}{I^{\prime} R}$ or $V=\frac{e i}{I^{\prime}}$. Finally, if the voltage of the battery in the external circuit is altered so that unit current is introduced into the lead, $I^{\prime}$ becomes 1 , and the final equation (5) is $V=e i$. This is the basic expression in the lead-field concept, and it states that the voltage in any lead due to an electromotive element in the heart equals the product of this element $e$ and the current that passes through the element if unit current is introduced into the lead. This idea may be extended to all elements of electromotive force in the heart. Thus in

$$
V=e_{1} i_{1}+e_{2} i_{2}+e_{3} i_{3}+\cdots
$$

* Wilson made this clear and concise translation from Helmholtz's original paper." 
where $V$ is the open-circuit voltage of a lead, the $e$ 's are the potential differences of the electromotive-force elements, and the $i$ 's are the currents passing through the elements when a unit current is introduced into a lead.

If unit current is introduced into the two electrodes of any lead, currents will flow through all parts of the body, including the heart; since, at any point in the body, the current will have a certain direction and magnitude, it may be considered as a vector field and be represented by $\vec{J}$, where the magnitude of $\vec{J}$ is measured in terms of current per unit area at every point. If the electromotive surfaces considered in the previous paragraph are very small the current field will be uniform over the whole element, and the total current passing through it will be the product of the component of the field perpendicular to the surface of the element and its area. By the fundamental equation (5) of the lead field, $V=e i$, the voltage produced in the lead by this element will be the product of this total current and the potential difference of the element. If we now define the electromotive vector $\vec{e}$ of any small element of electromotive surface as a vector that points in the direction faced by the positive side of the element, and that has a magnitude equal to the potential difference across the element multiplied by its area, then $V=\vec{J} \cdot \vec{e}$ or $V=J_{x} e_{x}+J_{y} e_{y}$ $+J_{z} e_{z}$. It will be observed that these equations for the lead voltage are the same, except for some difference in the symbols employed, as those relating to the lead vector, and it is clear that the lead field for any point in the heart is identical to the lead vector for that point. Equation 6, above, may now be written

$$
V=\vec{J}_{1} \cdot \vec{e}_{1}+\vec{J}_{2} \cdot \vec{e}_{2}+\vec{J}_{3} \cdot \vec{e}_{3}+\cdots
$$

and thus the essentially algebraic lead vector may be replaced by the more geometric and physical concept of the lead field.

It must be made clear at this point that the lead field is determined by the currents that flow through the body (including the heart). When an external battery of proper size is connected to the electrodes of any lead, however, this does not in any way alter the basic fact that voltages originating in the heart are responsible for electrocardiograms obtained when a suitable recording instrument is connected to those electrodes. Further, it must be emphasized that the part of the lead field passing through the heart is the only portion that is important in electrocardiography. The lead field is actually a device that helps in understanding the behavior of any lead and the design of new and better ones. The following illustrations should aid in making its value clear.

In FIGURE 9A the approximate lead field for lead 1 is illustrated. Here the heart is represented by the shaded area, and it will be observed that the current field produced by the external battery, that is, the lead field, passes in a generally transverse direction through the heart. This means that lead 1 depicts fairly well transverse components of voltages arising in the heart. This should be clear from the previous discussion of the nature of the lead field, but consideration of FIGURE 9B may make this even more obvious. Here a single element of the lead field and 2 sources of voltage, or dipoles $A$ and $B$ in the 


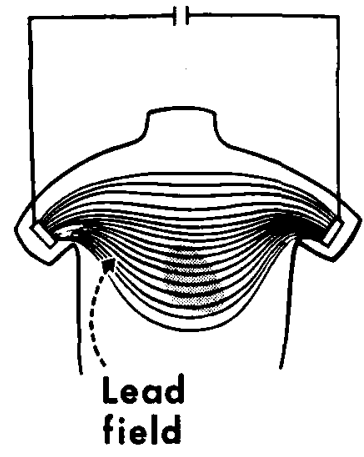

$A$

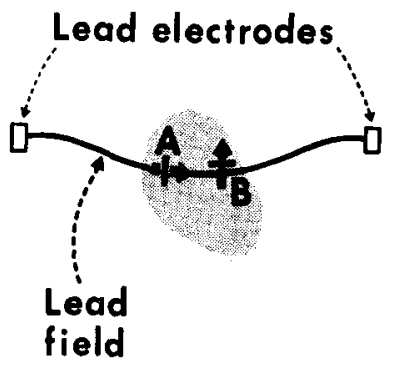

B

Figure 9. Reproduced by permission of F. A. Davis Co., Philadelphia, Pa.

heart, both located on this element, are shown. Source $A$ is oriented with its positive and negative poles parallel to the lead field, and it contributes in the greatest possible degree to the lead voltage. Source $B$, on the other hand, is oriented with the positive and negative poles at right angles to the lead field, and it contributes nothing to the lead voltage.

The implications of the above should be clear. Although it is not possible to determine the exact path of the lead field through the myocardium, fortunately intuition and common sense enable us to establish its approximate course through the heart by the use of simple spatial relationships between the locations of electrodes and the heart. The lead field thus provides a simple and powerful tool not only to explain how any type of lead will function but, even more important, to indicate what types of electrodes or electrode systems should be employed to obtain leads that are ideal for specific purposes.

In FIGURE $10 \mathrm{~A}$ we see a representation of the lead field for lead $\mathrm{a} V_{F}$ obtained from a fluid mapper as described by McFee et al. ${ }^{16}$ It is a hydraulic analog to the electrical situation in the human subject for the lead mentioned. Here water enters the orifice in the left leg, flows upward in a shallow space, and passes out in equal amounts through orifices in the two arms. Paths of fluid flow are visualized by small crystals of soluble dye, and the flow of currents (the lead field) through the human body would be similar if a battery were connected to the terminals of lead $a V_{F}$. It will be observed that the field is reasonably uniform and nearly vertical in direction. This suggests that this would be a satisfactory lead for obtaining the vertical component of cardiac voltages.

The ideal unipolar lead, from the lead-field standpoint, is one in which the field radiates symmetrically in all directions from the exploring electrode to the indifferent electrode (at infinity). The field obtained with a fluid mapper set up to represent a unipolar chest lead employing the central terminal is shown in FIGURE 10B. Here fluid passed into the model in equal amounts through orifices in the two arms and left leg and out through an opening over the cardiac area. This nearly perfect field may be contrasted to the one obtained for a CR chest lead (FIGURE 10C). Here fluid (or electricity) passes 


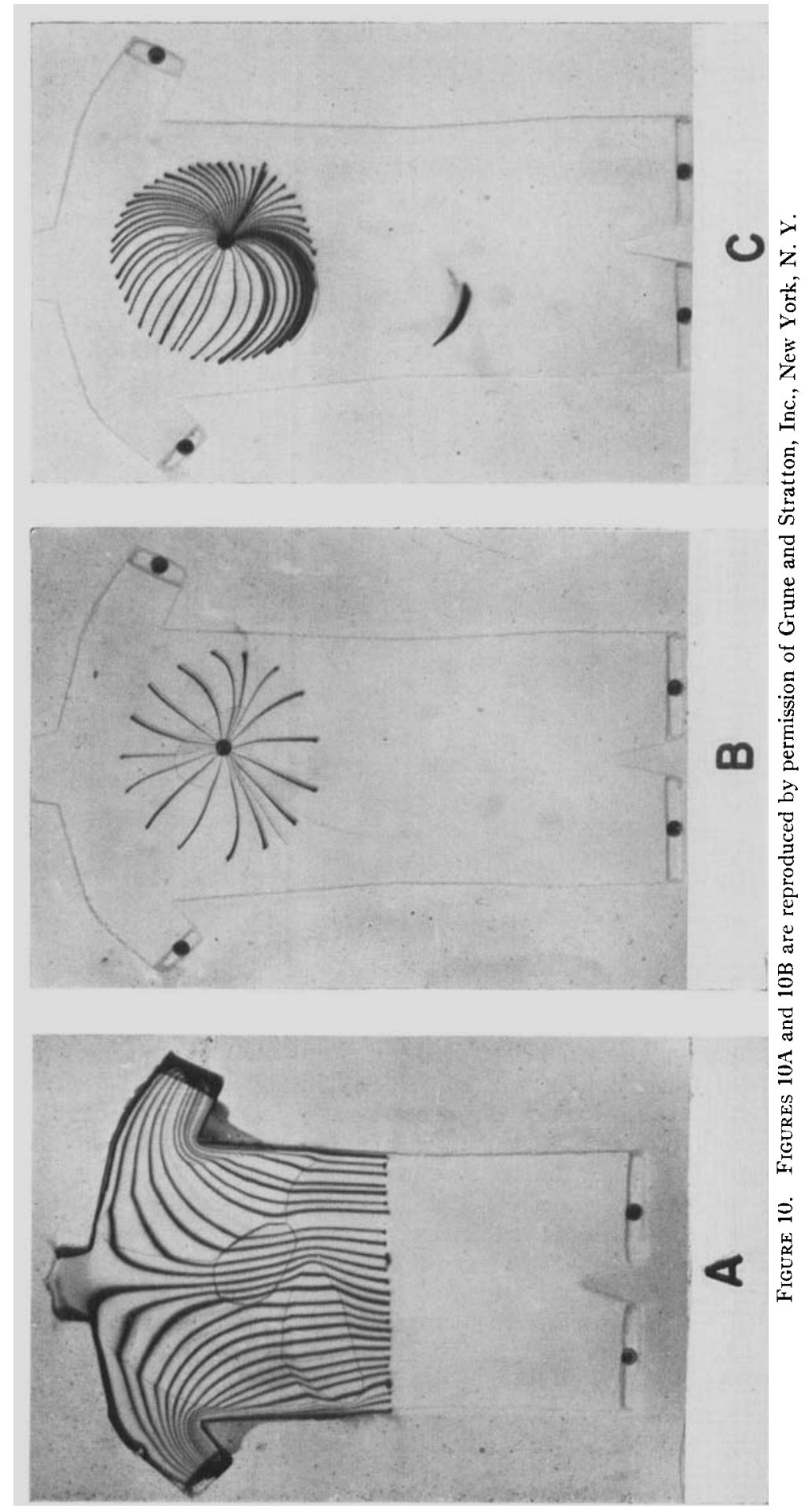



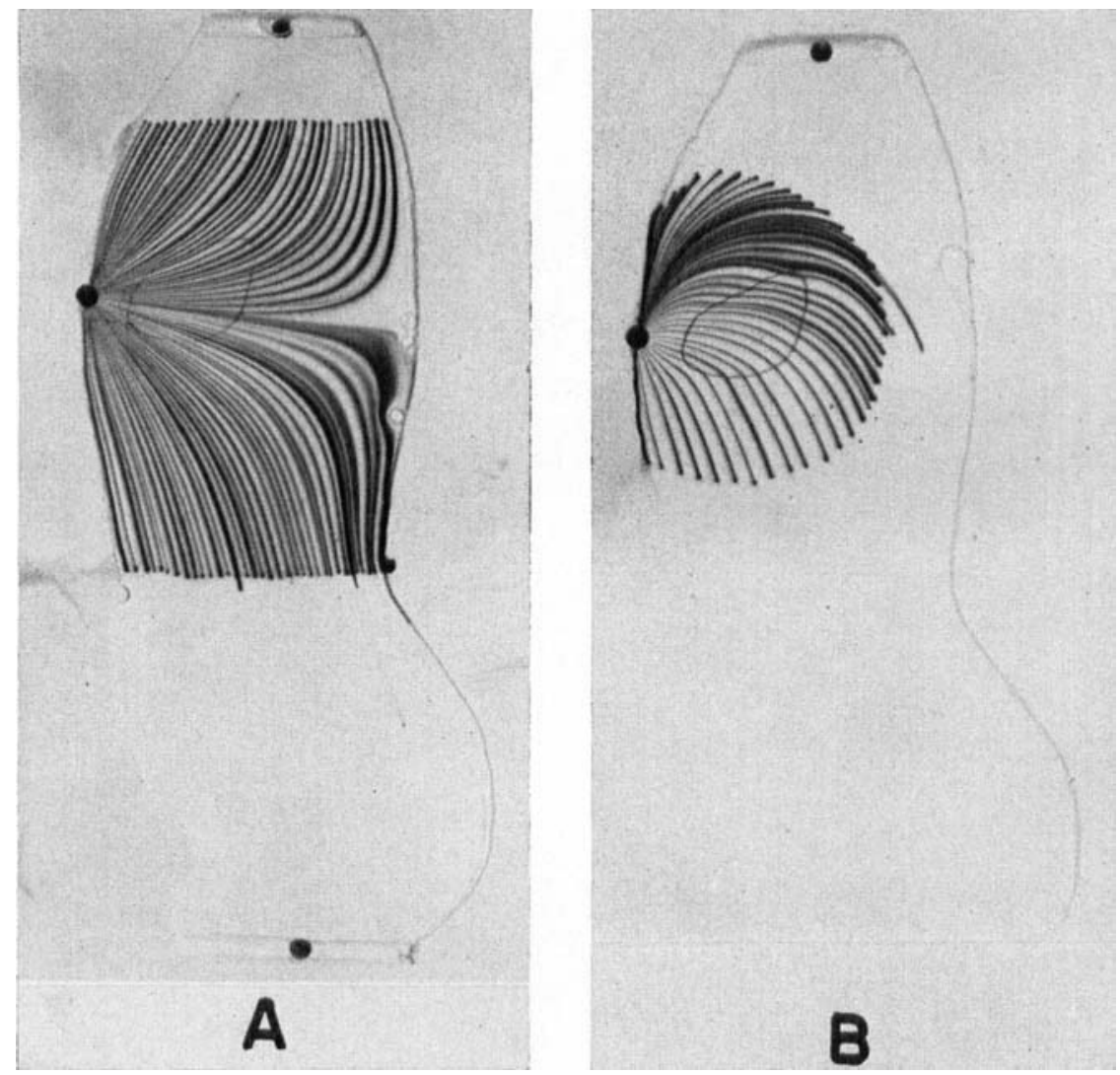

FIGURE 11

from a single orifice (or electrode) in the right arm and out through the aperture (or exploring electrode) over the heart. Figures 11A and 11B, respectively, illustrate sagittal views of the fields obtained with a chest lead employing the central terminal and the same chest lead where the leg was used as the indifferent electrode (CF lead). It will be noticed that in the former the field radiates in quite symmetrical fashion from the exploring electrode, and that there is concentration of the field in the anterior parts of the heart. The latter must mean that leads of this kind are more sensitive to voltages on the anterior than on the posterior aspect of the heart.

I wish now to emphasize a point that should by now be obvious. The leadfield concept makes clear the type of field that must exist in the heart if a lead is to be ideal for vectorcardiography. Thus, for the best possible transverse, vertical, and sagittal components of the cardiac E.M.F.s, the fields (associated with the leads employed) must be like those shown in FIGURE 12, $a, b$, and $c$, respectively. The type of electrode system required to obtain a good sagittal 
Frontal view

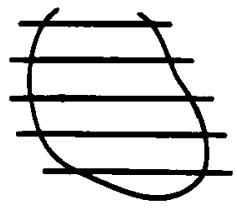

6

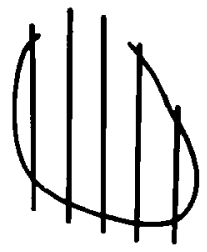

b
Lateral view

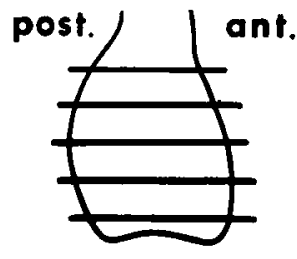

c

Figure 12. Reproduced by permission of F. A. Davis Co., Philadelphia, Pa.

component and its associated lead field are shown in diagrams $a$ and $b$, respectively, of FIGURE 13B. Several small electrodes, each connected through large equal resistances to a common electrode, are placed over the precordial areas anteriorly and posteriorly. The number of electrodes necessary to ensure that the lead field will be satisfactory will vary somewhat with heart size and with other factors. At the moment, it seems likely that a minimum of fifteen electrodes well distributed over the precordium and a somewhat fewer number posteriorly should be provided. The smaller the number of electrodes used, especially anteriorly, the more critical their placement will be. The advantage of the multiple-electrode grid arrangement for obtaining the sagittal component of cardiac E.M.F.s is shown very clearly in FIGURE 13A, where the lead field for this system is contrasted with those that exist with the so-called cube and the tetrahedron arrangements. Although the lead field for the latter has an average anterior-posterior direction, there is considerable curvature of the field, indicating that lead $V_{B}$ (employed for the tetrahedron system) is sensitive to vertical as well as to sagittal components of the heart voltages. Furthermore, this lead is more sensitive to voltages on the posterior than on the anterior aspect of the heart. The lead field for leads employed with the cube arrangement shows marked curvature; when it is remembered that the electrodes for this lead are located on the lower chest near the right anterior and

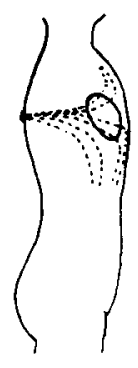

Frowetron

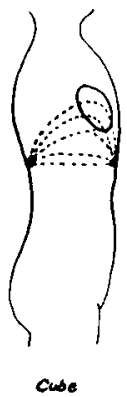

A

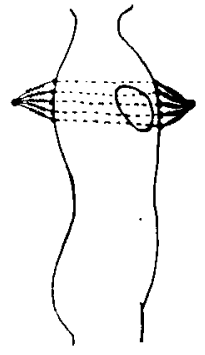

Mursipe elenow onis

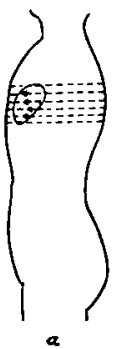

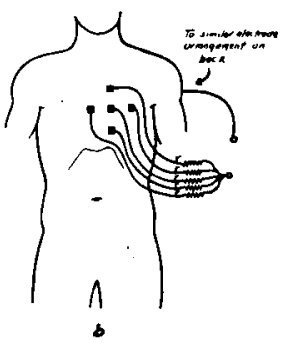

B

FIGCRE 13. Methods of obtaining a good sagittal component. (FIGURE 13A is reproduced by permission of Grune and Stratton, Inc., New York, N. Y.) 

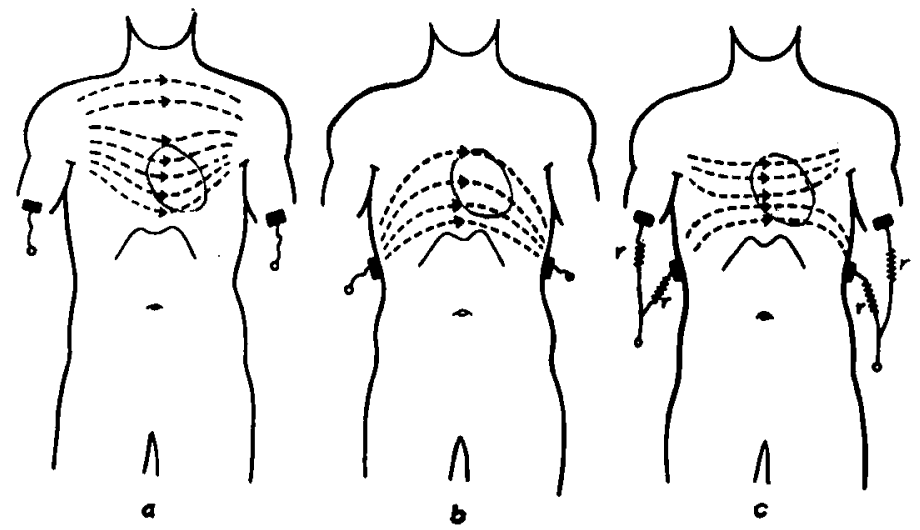

Figure 14. Reproduced by permission of Grune \& Stratton, Inc., New York, N. Y.

posterior axillary lines, it will be clear that this arrangement records transverse and vertical components of cardiac voltages, as well as sagittal components.

Finally, in FIGURE 14, a simple arrangement for improving the behavior of lead 1, if it is to be used for the transverse component of a vectorcardiogram, is shown. Thus, although the lead field for lead 1 alone (FIGURE 14a) has a general transverse direction, the curvature, particularly near the apex, impairs the accuracy of the lead for the purpose at hand. If, however, a lead with electrodes in the midaxillae slightly below the level of the heart (FIGURE 14b) is combined through large resistances with lead 1 (FIGURE 14c), the opposite curvature of the fields in $a$ and $b$ will tend to cancel each other, giving a resultant field with a more purely transverse direction.

This brief discussion of the lead field is incomplete in many respects, but it will furnish, I hope, some indication of its power and simplicity.

In summary, I have tried to outline some of the basic considerations relating to the distribution of currents and potentials in homogeneous volume conductors, including the use made of some of these matters by Einthoven, Wilson, and others. The lead vector of Burger and van Milaan has been briefly described, and a somewhat more detailed discussion of the lead field has been given.

\section{Acknowledgments}

I wish to make it clear that the lead-field concept and its development have been almost entirely the work of Richard McFee, and to him must go the credit for this fine contribution. I am also indebted to several of my present and former associates for helping in the collection of some of the material presented in this paper. These include Ernest W. Reynolds, Jr., Robert M. Stow, Jerome F. Cordes, and Park W. Willis, III. My debt to Frank N. Wilson in so many ways is so great that I can find no words to express it.

\section{References}

1. Wilson, F. N., A. G. Macleod \& P. S. Barker. 1933. The distribution of the currents of action and injury displayed by heart muscle and other excitable tissues. Univ. Mich. Press. Ann, Arbor, Mich. 


\section{Johnston: Homogeneous Volume Conductors}

2. CraIb, W. H. 1927. A study of the electrical field surrounding active heart muscle. Heart. 14: 71.

3. Crair, W. H. 1928. A sturly of the electrical field surrounding skeletal muscle. J. Physiol. 66: 49 .

4. Craib, W. H. 1930. 'The electrocardiogram. Med. Research (ouncil Brit. Spec. Rep). Series No. 147.

5. Wilson, F. N., S. W. Wishart \& G. R. Herrmann. 1926. Factors influencing the distribution of potential differences produced by heart beat at the surface of the body. Proc. Soc. Exptl. Biol. Mer. 23: 276.

6. Wilson, F. N. 1930. Distribution of the potential differences produced by the heart beat within the body and its surface. Am. Heart J. 5: 599.

7. Wilson, F. N., F. D. Johnston, A. G. Macleod \& P. S. Barker. 1934. Electrocardiograms that represent the potential variations of a single electrode. Am. Heart J. 9: 447.

8. GoLpberger, E. 1942. A simple indifferent electrocardiographic electrode of zero potential and a technique of obtaining augmented unipolar extremity leads. Am. Heart J. 23: 483 .

9. Burger, H. C. \& J. B. van Milaan. 1946. Heart vector and leads. I. Brit. Heart J. 8: 157 .

10. Burger, H. C. \& J. B. van Milaan. 1947. Heart vector and leads. II. Brit. Heart J. 9: 154 .

11. MCFEE, R. \& F. D. Johnsron. 1953. Electrocardiographic leads. I. Introduction. Circulation. 8: 554.

12. McFeE, R. \& F. D. Johnston. 1954. Electrocardiographic leads. II. Analysis. Circulation. 9: 255 .

13. MCFEE, R. \& F. D. Johnston. 1954. Electrocardiographic leads. III. Synthesis. Circulation. 9: 868 .

14. LepeschkIN, E. 1951. Modern Electrocardiography. Williams \& Wilkins. Baltimore, $\mathrm{Md}$.

15. Helmholtz, H. 1853. Über einige Gesetze der Vertheilung elektrischer Ströme in Körperlischen Leitern mit Anwendung auf die thierisch elektrischen Versuche. Ann. Physiol. Chem. 29(3): 222.

16. MCFEE, R., R. M. Stow \& F. D. Johnston. 1952. Graphic representation of electrocardiographic leads by means of fluid mappers. Circulation. 4: 21. 